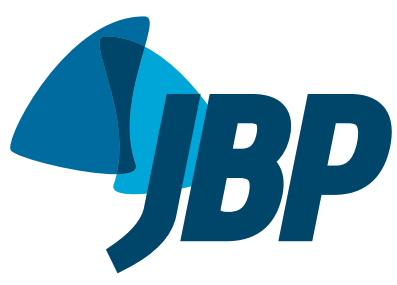

1. Divisão Respiratória, Universidade Federal de São Paulo - UNIFESP São Paulo (SP) Brasil.

Submitted: 13 June 2016.

Accepted: 12 September 2016

Study carried out in the Divisão

Respiratória, Universidade Federal de São Paulo - UNIFESP - São Paulo (SP) Brasil.

\section{Family caregiver burden: the burden of caring for lung cancer patients according to the cancer stage and patient quality of life}

\author{
Eliana Lourenço Borges ${ }^{1}$, Juliana Franceschini ${ }^{1}$, Luiza Helena Degani Costa1,
} Ana Luisa Godoy Fernandes ${ }^{1}$, Sérgio Jamnik¹, Ilka Lopes Santoro

\begin{abstract}
Objective: Patients with lung cancer experience different feelings and reactions, based on their family, social, cultural, and religious backgrounds, which are a source of great distress, not only for the patients but also for their family caregivers. This study aimed to evaluate the impact that lung cancer stage and quality of life (QoL) of lung cancer patients have on caregiver burden. Methods: This was a prospective cross-sectional study. Consecutive patient-caregiver dyads were selected and asked to complete the Hospital Anxiety and Depression Scale and the Medical Outcomes Study 36-item ShortForm Health Survey (SF-36). Family caregivers also completed the Caregiver Burden Scale. Group-based modeling was used in order to identify patients with early- or advanced-stage cancer (IA to IIIA vs. IIIB to IV) plus non-impaired or impaired QoL (SF36 total score $>50$ vs. $\leq 50$ ). Patient-caregiver dyads were stratified into four groups: early-stage cancer+non-impaired QoL; advanced-stage cancer+non-impaired CoL; early-stage cancer+impaired QoL; and advanced-stage cancer+impaired OoL. Results: We included 91 patient-caregiver dyads. The majority of the patients were male and heavy smokers. Family caregivers were younger and predominantly female. The burden, QoL, level of anxiety, and level of depression of caregivers were more affected by the QoL of the patients than by their lung cancer stage. The family caregivers of the patients with impaired QoL showed a higher median burden than did those of the patients with non-impaired QoL, regardless of disease stage. Conclusions: Caregiver burden is more affected by patient QoL than by lung cancer stage.
\end{abstract}

Keywords: Lung neoplasms; Quality of life; Caregivers; Anxiety; Depression; Cost of illness.

\section{INTRODUCTION}

Advances in diagnostic and treatment strategies for lung cancer (LC), together with an increasing, aging population have resulted in a shift from impatient to outpatient treatment, underscoring the importance of family caregivers. ${ }^{(1-3)}$ Despite the advances in treatment, patients faced with a diagnosis of LC experience different feelings and reactions, based on their family backgrounds, which are undoubtedly a source of great distress not only for the patient but also for family caregivers. ${ }^{(3)}$

Family caregivers are usually relatives, partners, or close friends who have a significant personal relationship with the patient and provide a broad range of assistance for the person with a chronic or disabling condition, such as LC. ${ }^{(2)}$ Family caregivers are expected to assist the patients in every aspect of their lives, which could range from helping with basic activities of daily living to providing emotional, social, and financial support. ${ }^{(4,5)}$ In this setting, the burdens of family caregiving may include not only physical tasks but also emotional distress, since caregivers tend to neglect their own needs on behalf of the patient. ${ }^{(6)}$

Despite the increasing attention given to caregivers and families in the cancer literature, some health professionals still remain unaware of the fact that patients and caregivers have an interdependent relationship, in terms of their quality of life (QoL), and therefore fail to address the needs of caregivers as a part of the therapeutic strategy. ${ }^{(7,8)}$ In addition, much of the current knowledge about the experience of family caregivers derives from clinical impressions rather than from research.

It has been suggested that the burden of family caregivers of cancer patients might vary according to the illness stage and could depend on factors related to the patient condition. Although some studies have addressed QoL and the burden of family caregivers of patients with LC, only a few studies have related those aspects to the perception that patients have of their own health-related QoL. $(2,3,6)$ Therefore, the objective of the present study was to evaluate the impact that LC stage and LC patient QoL have on the family caregiver burden.

\section{METHODS}

\section{Subjects and study design}

We performed a prospective cross-sectional study in order to identify the features of family caregiver burden. 
Consecutive patient-caregiver dyads were selected from the Oncology Outpatient Clinic at the University Hospital of the Universidade Federal de São Paulo, located in the city of São Paulo, Brazil, when they came for their routine evaluation prior to starting treatment. We included only those patient-caregiver dyads in which the patient and the family caregiver both agreed to participate. The study was approved by the local institutional review board, and all participants gave written informed consent.

Eligible patients had a clinical and histological diagnosis of LC and a Mini-Mental State Examination ${ }^{(9)}$ score of 20 or higher. In addition, family caregivers were considered eligible if they were over 18 years of age, had a Mini-Mental State Examination score of 20 or higher, ${ }^{(9)}$ and were regarded by the patient as his or her primary caregiver (the relative most involved with his or her current care).

\section{Cancer stage/QoL grouping}

In order to compare the variables of the patients and of their family caregivers, the sample was stratified. For this process, we used the disease stage and the QoL of the patients, the latter being assessed with the Medical Outcomes Study 36-item Short-Form Health Survey (SF-36). ${ }^{(10)}$ Group-based modeling was used in order to identify patients with early- or advanced-stage cancer (IA to IIIA vs. IIIB to IV) plus non-impaired or impaired QoL (an SF-36 total score of > 50 vs. $\leq 50$ ). Therefore, patient-caregiver dyads were stratified into four groups (Figure 1):

- Early-stage cancer plus non-impaired QoL

- Advanced-stage cancer plus non-impaired QoL

- Early-stage cancer plus impaired QoL

- Advanced-stage cancer plus impaired QoL

\section{Data collection and procedures}

A structured form was filled out regarding sociodemographic characteristics and the Karnofsky Performance Status (KPS) scale results of the patients. The characteristics and the disease-related variables studied included gender, age (in years), smoking status (never/ever-smoker), histological cancer type (squamous cell, adenocarcinoma, or other), LC stage, ${ }^{(11)}$ and treatment status (yes/no). Characteristics of family caregivers included gender, age (in years), smoking status (never/ever-smoker), educational background, hours/day providing direct care, and relationship with the patient (child, spouse, or other). Patient-caregiver dyads were asked to complete the Hospital Anxiety and Depression Scale (HADS) ${ }^{(10)}$ and the SF-36 in separate locations, so that information and responses would be neither shared nor influenced by the other subject. In addition, family caregivers also completed the Caregiver Burden Scale (CBS). ${ }^{(13)}$

Psychological distress was assessed by the HADS, which is a 14-item instrument with two dimensions (7 items each) that assesses anxiety and depression symptoms. Each item is scored on a 4-point scale from 0 (not present) to 3 (considerable), adding up to a maximum possible score of 21 . $^{(12)}$ The HADS has been validated as a screening instrument for use in Brazil. (14) The probability of subjects experiencing anxiety or depression was established according to predetermined thresholds for each of the dimensions: a score < 8 was considered indicative of an improbable diagnosis; scores between 8 and 11 were considered indicative of a possible diagnosis; and scores $>11$ were considered indicative of a highly likely diagnosis of depression, anxiety, or both. ${ }^{(15)}$ For statistical analysis, the scores were transformed into a dichotomous variable: a likely $(\geq 8)$ or an unlikely $(<8)$ diagnosis of anxiety or depression.

Health-related QoL was assessed by the SF-36, which has previously been translated into Portuguese and validated for use in Brazil.(10) It consists of 11 items with a total of 36 different possible answers, which are grouped into eight main domains: functional capacity, role-physical, bodily pain, general health, vitality, social functioning, role-emotional, and mental health. ${ }^{(16)}$ For each dimension, questions are coded, the scores are summed, and the overall score is transformed into a score from 0 (the worst QoL) to 100 points (the best QoL). Although there is no agreement about the optimal cut-off point to use, a review of the individual SF-36 total score accuracy found that the best trade-off between sensitivity and specificity was achieved using 50 as a cut-off point. Therefore, an SF-36 total score $\leq 50$ would identify impaired QoL.

The CBS version validated for use in Brazil was applied in order to assess the family caregiver burden associated with the functional and behavioral disability of the patient. ${ }^{(13)}$ It is a self-report 22-item scale with five dimensions: general tension, isolation, disappointment, emotional involvement, and environment. This tool is measured on a 4-point Likert scale that ranges from

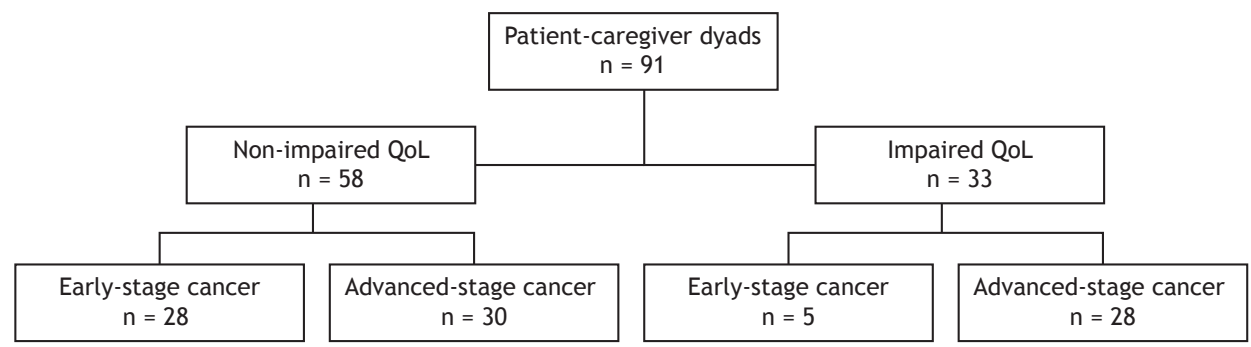

Figure 1. Algorithm for patient-caregiver dyads in the group-based modeling according to disease stage and patient quality of life $(\mathrm{Q} O L)$ stratification. 
1 (never) to 4 (nearly always), on which a high total score represents a high burden. The caregiver burden was stratified into two levels: minimal (from 0 to 20 points in the total score) and considerable (from 21 to 60 points). ${ }^{(17)}$

\section{Statistical analysis}

For the sample size calculation, we took into account the fact that one study reported that $16 \%$ of the caregivers for COPD patients had a CBS total score over 26 points. ${ }^{(18)}$ Assuming that $36 \%$ of the caregivers for LC patients had similar results in the CBS, 82 patient-caregiver dyads would be required for a type I error of $5 \%$ and a power of $80 \%$.

For statistical analyses, we stratified the patient-caregiver dyads into four groups according to LC stage and QoL of the patients. Descriptive analyses were conducted for each group in order to explore the relations among all demographic, psychological, and clinical variables. The chi-square test or Fisher's exact test was used for categorical variables. Continuous variables were analyzed using the Student's t-test, the Kruskal-Wallis test, or the Mann-Whitney test, depending on distribution of the variable.

Significance levels were set at $p<0.05$, and data were analyzed using the IBM SPSS Statistics software package, version 19 (IBM Corporation, Armonk, NY, USA).

\section{RESULTS}

\section{Participant characteristics}

A total of 91 patient-caregiver dyads met the inclusion criteria. The majority of the patients (56\%) were men, and a great majority (77\%) were heavy smokers (median smoking history, 50 pack/years [interquartile range: $30-70]$ ). Although $64 \%$ of the patients had locally advanced or metastatic disease, they showed a good level of performance status (median KPS score $=90$ ). The predominant histological type (in 52\%) was adenocarcinoma.

Overall, the family caregivers were younger than the patients ( $47.6 \pm 13.2$ years vs. $65.2 \pm 11.1$ years), predominantly female $(84 \%)$, and never-smokers $(61 \%)$. The majority $(63 \%)$ had a high level of education (over 12 years of schooling). According to the family caregivers themselves, nearly half of them were daughters of their patients. The median (interquartile range) of the duration of daily caregiving was $4 \mathrm{~h}(2-10 \mathrm{~h})$.

\section{Analyses of patient and family caregiver characteristics by group-based modeling}

Table 1 shows the characteristics of the patient-caregiver dyads, stratified into four groups: early-stage cancer+non-impaired QoL ( $\mathrm{n}=28)$; advanced-stage cancer+non-impaired QoL $(n=30)$; early-stage cancer+impaired QoL ( $n=5)$; and advanced-stage cancer+impaired QoL $(n=28)$.
Among the patients, the proportion of men was significantly greater in the early-stage cancer+non-impaired QoL group than in the other groups. The KPS score changed according to QoL and disease stage; it was significantly higher in the early-stage cancer+non-impaired QoL group than in the advanced-stage cancer+impaired QoL group. No differences were found among the four groups of patients regarding level of education, smoking status, histological type, or treatment status. In addition, the time that family caregivers spent providing direct care to the patients was significantly greater in the advanced-stage cancer+impaired QoL group than in the early-stage cancer+non-impaired QoL group. No differences were found among the four groups of family caregivers regarding gender, age, level of education, smoking status, or type of relationship between caregiver and patient.

Among the family caregivers of patients with advanced-stage cancer, those who took care of the patients with impaired QoL had a significantly higher caregiver burden, worse symptoms of anxiety/ depression, and worse QoL than did the caregivers of patients with non-impaired QoL (Figure 2). In addition, the caregivers of patients with early-stage cancer+non-impaired QoL had a lower level of caregiver burden than did those of patients with advanced-stage cancer+impaired QoL.

\section{DISCUSSION}

In this study, a multi-evaluation strategy was applied in order to measure caregiver burden by investigating QoL, anxiety, and depression from the perspectives of patients and family caregivers at the same time. The caregiver burden, as well as their QoL, anxiety, and depression, was more affected by the QoL of the patients than by the LC stage. In this sense, caregivers of patients with impaired QoL had a greater caregiver burden regardless of the disease stage. Therefore, the QoL of the patient has a significant impact on caregiver burden. ${ }^{(19,20)}$ Another way of interpreting these results is to acknowledge that stratifying patients by cancer stage (early and advanced) is an overly simplistic way of defining the patient-caregiver dyad situations. In the present study, the most important predictor for family caregiver burden was the SF-36 score of the patient. The relationship between the QoL of the patient and the caregiver burden reflects a vicious cycle. ${ }^{21,22)}$ Although many patients with impaired QoL need uninterrupted daily care, family caregivers are facing issues and tasks for which they have no qualifications or training. In addition, family caregivers have to deal with their own physical limitations and medical problems. This overwhelms the caregiver, who ends up providing insufficient help, which leads to further impairment of the QoL of the patient and increases his or her dependence. ${ }^{(23,24)}$ Furthermore, some authors have reported the presence of more intense anxiety symptoms in the caregivers of patients with advanced-stage cancer. ${ }^{(20,25)}$ Although increased caregiver anxiety tends to be associated with caregiver 
Table 1. Characteristics of the patients and caregivers according to the group-based modeling used in the study (disease stage and quality of life of the patients). ${ }^{\mathrm{a}}$

\begin{tabular}{|c|c|c|c|c|c|}
\hline \multirow[t]{2}{*}{ Variable } & \multicolumn{4}{|c|}{ Patient groups } & \multirow[t]{2}{*}{$\mathbf{p}^{*}$} \\
\hline & $\begin{array}{c}\text { ES + NI-QoL } \\
n=28\end{array}$ & $\begin{array}{c}\mathrm{AS}+\mathrm{NI}-\mathrm{QoL} \\
\mathrm{n}=30\end{array}$ & $\begin{array}{c}E S+I-Q o L \\
n=5\end{array}$ & $\begin{array}{c}\text { AS + I-QoL } \\
n=28\end{array}$ & \\
\hline \multicolumn{6}{|l|}{ Patients } \\
\hline Male & $22(78)$ & $13(43)$ & $2(40)$ & $14(50)$ & 0.03 \\
\hline Age, years & $68.0 \pm 8.2$ & $64.2 \pm 11.8$ & $62.6 \pm 12.9$ & $64.1 \pm 12.6$ & NS \\
\hline KPS score & $91.4 \pm 6.5$ & $85.7 \pm 7.3$ & $80.0 \pm 18.7$ & $80.7 \pm 11.2$ & $<0.001$ \\
\hline Under treatment & $23(82)$ & $23(77)$ & $3(60)$ & $15(54)$ & 0.09 \\
\hline \multicolumn{6}{|l|}{ Level of education, years } \\
\hline$\leq 9$ & $22(79)$ & $19(63)$ & $4(100)$ & $24(86)$ & NS \\
\hline$>9$ & $6(21)$ & $11(37)$ & $0(0)$ & $4(14)$ & \\
\hline \multicolumn{6}{|l|}{ Smoking status } \\
\hline Never smoker & $3(11)$ & $6(20)$ & $2(40)$ & $10(36)$ & NS \\
\hline Ever smoker & $25(89)$ & $24(80)$ & $3(60)$ & $18(64)$ & \\
\hline Smoking history, pack-years & $50.5 \pm 36.3$ & $35.6 \pm 33.6$ & $52.8 \pm 61.2$ & $29.9 \pm 30.5$ & NS \\
\hline \multicolumn{6}{|l|}{ Histological type } \\
\hline Squamous cell & $10(36)$ & $10(33)$ & $2(40)$ & $8(28)$ & NS \\
\hline Adenocarcinoma & $14(50)$ & $16(54)$ & $1(20)$ & $16(57)$ & \\
\hline \multirow[t]{2}{*}{ Others } & $4(14)$ & $4(13)$ & $2(40)$ & $4(15)$ & \\
\hline & \multicolumn{4}{|c|}{ Patient groups } & \\
\hline Caregivers & ES + NI-QoL & AS + NI-QoL & ES + I-QoL & AS + I-QoL & \\
\hline Female & $25(89)$ & $24(80)$ & $4(80)$ & $23(82)$ & NS \\
\hline Age, years & $48.8 \pm 9.5$ & $45.3 \pm 14.7$ & $39.8 \pm 10.8$ & $50.4 \pm 14.7$ & NS \\
\hline \multicolumn{6}{|l|}{ Level of education, years } \\
\hline$\leq 9$ & $10(36)$ & $10(33)$ & $0(0)$ & $13(47)$ & NS \\
\hline$>9$ & $18(64)$ & $20(67)$ & $5(100)$ & $15(53)$ & \\
\hline \multicolumn{6}{|l|}{ Smoking status } \\
\hline Never smoker & $15(54)$ & $20(67)$ & $3(60)$ & $18(64)$ & NS \\
\hline Ever smoker & $13(46)$ & $10(33)$ & $2(40)$ & $10(36)$ & \\
\hline Smoking history, pack-years & $7.6 \pm 15.4$ & $9.4 \pm 17.3$ & $6.2 \pm 3.3$ & $4.9 \pm 11.0$ & NS \\
\hline \multicolumn{6}{|c|}{ Relationship between caregiver and patient } \\
\hline Child & $16(57)$ & $13(43)$ & $4(80)$ & $12(44)$ & NS \\
\hline Spouse & $10(36)$ & $10(33)$ & $0(0)$ & $8(28)$ & \\
\hline Sibling & $2(7)$ & $7(24)$ & $1(20)$ & $8(28)$ & \\
\hline \multicolumn{6}{|l|}{ Direct caregiving, h/day } \\
\hline$<4$ & $24(86)$ & $14(47)$ & $3(60)$ & $12(43)$ & 0.004 \\
\hline$\geq 4$ & $4(14)$ & $16(53)$ & $2(40)$ & $16(57)$ & \\
\hline
\end{tabular}

ES: early-stage (cancer); NI: non-impaired; QoL: quality of life; AS: advanced-stage (cancer); I: impaired; KPS: Karnofsky Performance Status; and NS: not significant. aValues expressed in $\mathrm{n}(\%)$ or mean \pm SD. ${ }^{*}$ Chi-square test and one-way ANOVA.

perception of the disease progression, ${ }^{(8)}$ in the present study, the level of caregiver anxiety was not a risk factor for increased caregiver burden. This might be related to the tendency of caregivers to underestimate their perception of anxiety. It is worth noting the culture among Brazilian caregivers; we believe that their major concern is to meet the needs and expectations of the patient at the expense of their own needs.

It is also important to highlight the fact that the proportion of smokers and their smoking history among family caregivers of LC patients are almost 10 times smaller/lower than those observed among the patients in the dyads. This result may be related to the increasingly intensive antismoking policies implemented in the past 10 years in Brazil.
In the present study, nearly half of the family caregivers was taking care of the patients for over $4 \mathrm{~h} /$ day (28 h/week), which is higher than the $24.4 \mathrm{~h} /$ week reported by the National Alliance for Caregiving. ${ }^{(26)}$ As discussed above, family caregivers reported a greater caregiving burden when the QoL of the patients worsened; conversely, the number of hours/day for providing direct caregiving was greater among caregivers whose patients had advanced-stage cancer, regardless of the QoL of the patient. Cancer treatment is known to be a arduous path, comprising frequent surgical or clinical interventions and multiple hospitalizations, as well as overwhelming physical and emotional stress. ${ }^{(19)}$ Consequently, regardless of how fit and independent the patient is at the time of the 

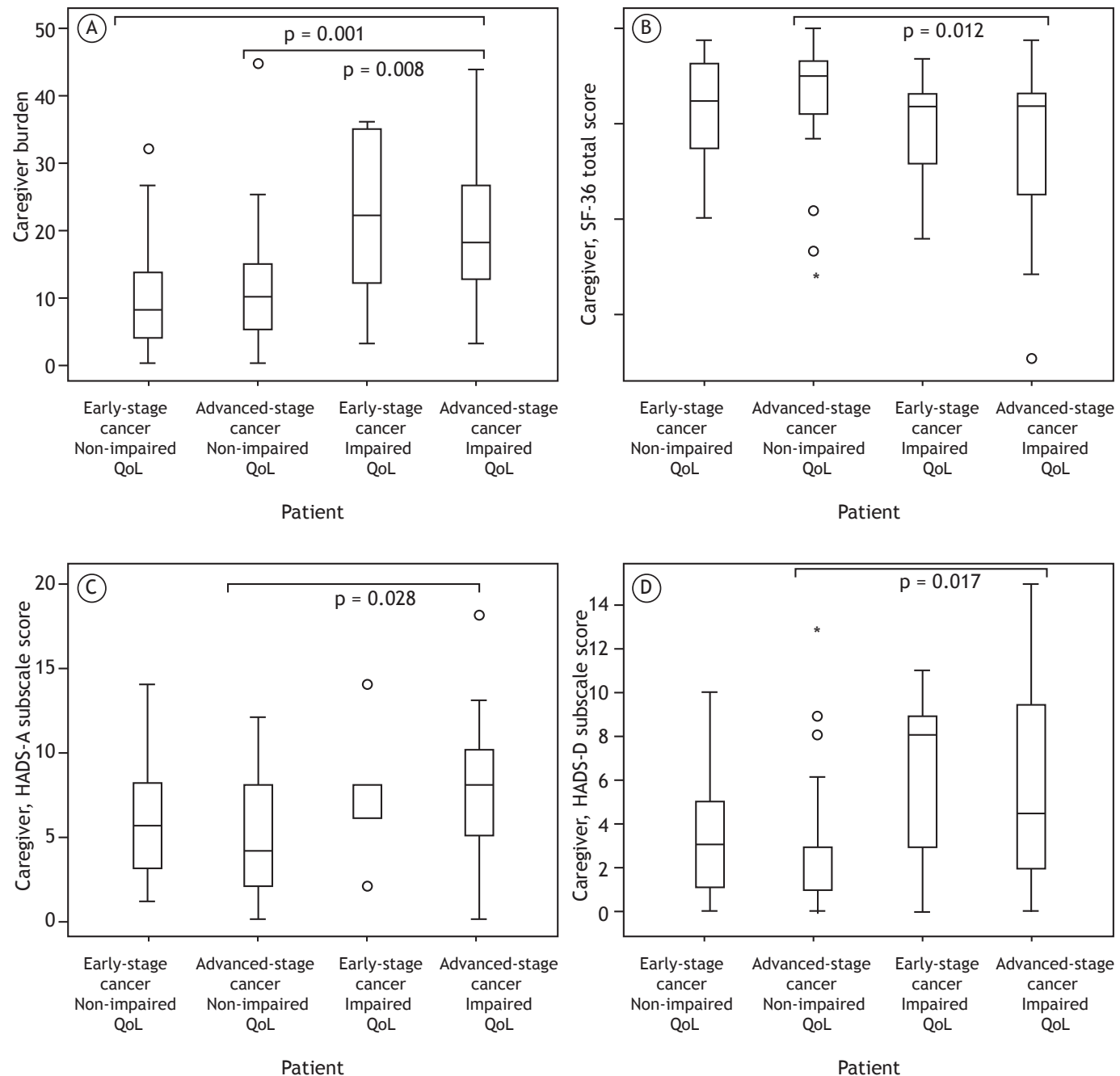

Figure 2. Distribution of caregiver burden (in A), SF-36 total score of caregivers (in B), HADS-A subscale score of caregivers (in C), and HADS-D subscale score of caregivers (in D) according to the group-based modeling adopted in the study. QoL: quality of life; SF-36: Medical Outcomes Study 36-item Short-Form Health Survey; HADS: Hospital Anxiety and Depression Scale; A: anxiety; and D: depression. *Kruskal-Wallis and post-hoc Dunn tests.

diagnosis, he or she will eventually need a caregiver to help him or her throughout the illness trajectory. ${ }^{(21,27,28)}$

A traditional Brazilian value has been that families take responsibility for the care of their elderly members. Although this situation implies lower costs, supportive policies and multidisciplinary palliative care services do not provide enough support for those families. In addition, the great majority of family caregivers are unprepared to provide care, having little knowledge about the disease and its course. ${ }^{(29)}$

Some limitations of our study should be noted. First, it was a cross-sectional study, and, consequently, caution should be used when interpreting the results. The study design prevented us from determining the stability of the family caregiver involvement over time. Further studies are needed in order to investigate the long-term effect that patient QoL has on family caregiver burden. Second, although a group of family members might be sharing tasks and making decisions regarding treatment, which might have some implications in caregiver burden, we did not consider multiple caregivers in the present analysis. Despite these limitations, our study has relevance for the clinical perspective that the family caregiver can assume more autonomy instead of being a passive participant in the caregiving process framework. Interaction among physicians, families, and patients, based on effective communication, is crucial in order to improve QoL and reduce the caregiver burden.

Future research regarding practical, behavioral, and self-care skills is encouraged so that patients and caregivers can successfully cope with LC in an easier way. It is crucial that efforts should be developed in order to relieve the caregiver burden by creating a framework to help promote supportive relationships in which the patient and the caregiver will both benefit. Therefore, we intend to design a supportive, personalized multidisciplinary approach in order to strengthen caregiver confidence in giving care, which will increase the quality of caregiving. Because there 
is a dynamic relationship between the burden and the satisfaction of caregiving, this framework can provide caregivers with realistic expectations.
In summary, the results in our study suggest that caregiver burden is more affected by the QoL of the patients than by their LC stage.

\section{REFERENCES}

1. Siegel R, Ma J, Zou Z, Jemal A. Cancer statistics, 2014. CA Cancer J Clin. 2014;64(1):9-29. http://dx.doi.org/10.3322/caac.21208

2. Blum K, Sherman DW. Understanding the experience of caregivers: a focus on transitions. Semin Oncol Nurs. 2010;26(4):243-58. http:// dx.doi.org/10.1016/j.soncn.2010.08.005

3. Hirdes JP, Freeman S, Smith TF, Stolee P. Predictors of caregiver distress among palliative home care clients in Ontario: evidence based on the interRAl Palliative Care. Palliat Support Care. 2012;10(3):155-63. http://dx.doi.org/10.1017/S1478951511000824

4. Adelman R, Tmanova LL, Delgado D, Dion S, Lachs MS. Caregiver burden: a clinical review. JAMA. 2014;311(10):1052-60. http://dx.doi. org/10.1001/jama.2014.304

5. Schubart JR, Kinzie MB, Farace E. Caring for the brain tumor patient: family caregiver burden and unmet needs. Neuro Oncol. 2008;10(1):61-72. http://dx.doi.org/10.1215/15228517-2007-040

6. Stajduhar KI. Burdens of family caregiving at the end of life. Clin Invest Med. 2013;36(3):E121-6

7. Grant M, Sun V, Fujinami R, Sidhu R, Otis-Green S, Juarez G, et al. Family caregiver burden, skills preparedness, and quality of life in non-small cell lung cancer. Oncol Nurs Forum. 2013;40(4):337-46. http://dx.doi.org/10.1188/13.ONF.337-346

8. Costa-Requena G, Cristófol R, Cañete J. Caregivers' morbidity in palliative care unit: predicting by gender, age, burden and selfesteem. Support Care Cancer. 2012;20(7):1465-70. http://dx.doi. org/10.1007/s00520-011-1233-6

9. Crum RM, Anthony JC, Bassett SS, Folstein MF. Population-based norms for the Mini-Mental State Examination by age and educational level. JAMA. 1993;269(18):2386-91. http://dx.doi.org/10.1001/ jama.1993.03500180078038

10. Cicconelli RM, Ferraz MB, Santos W, Meinão I, Quaresma MR Tradução para a língua portuguesa e validação do questionário genérico de avaliação da qualidade de vida SF-36 (Brasil SF-36). Rev Bras Reumatol. 1999;39(3):143-50

11. Edge S, Byrd DR, Compton CC, Fritz AG, Greene FL, Trotti A, editors. AJCC Cancer Staging Manual. 7th edition. New York: SpringerVerlag; 2010

12. Bjelland I, Dahl AA, Haug $T$, Neckelmann D. The validity of the Hospital Anxiety and Depression Scale. An updated literature review. J Psychosom Res. 2002;52(2):69-77. http://dx.doi.org/10.1016/ S0022-3999(01)00296-3

13. Medeiros MM, Ferraz MB, Quaresma M, Menezes AP. Adaptation and validation of the caregiver burden scale to Brazilian cultural milieu [Article in Portuguese]. Rev Bras Reumatol. 1998;38(4):193-9.

14. Botega NJ, Bio MR, Zomignani MA, Garcia C Jr, Pereira WA. Mood disorders among inpatients in ambulatory and validation of the anxiety and depression scale HAD [Article in Portuguese]. Rev Saude Publica. 1995;29(5):355-63.

15. Price MA, Butow PN, Costa DS, King MT, Aldridge LJ, Fardell JE, et al. Prevalence and predictors of anxiety and depression in women with invasive ovarian cancer and their caregivers. Med J Aust. 2010;193(5 Suppl):S52-7.

16. Franceschini J, Santos AA, El Mouallem I, Jamnik S, Uehara
C, Fernandes $A L$, et al. Assessment of the quality of life of patients with lung cancer using the Medical Outcomes Study 36item Short-Form Health Survey [Article in Portuguese]. J Bras Pneumol. 2008;34(6):387-93. http://dx.doi.org/10.1590/S180637132008000600009

17. Elmstahl S, Malmberg B, Annerstedt L. Caregiver's burden of patients 3 years after stroke assessed by a novel caregiver burden scale. Arch Phys Med Rehabil. 1996;77(2):177-82. http://dx.doi. org/10.1016/S0003-9993(96)90164-1

18. Pinto RA, Holanda MA, Medeiros MM, Mota RM, Pereira ED Assessment of the burden of caregiving for patients with chronic obstructive pulmonary disease. Respir Med. 2007;101(11):2402-8. http://dx.doi.org/10.1016/j.rmed.2007.06.001

19. Palos GR, Mendoza TR, Liao KP, Anderson KO, Garcia-Gonzalez A, Hahn K, et al. Caregiver symptom burden: the risk of caring for an underserved patient with advanced cancer. Cancer. 2010;117(5):1070-9. http://dx.doi.org/10.1002/cncr.25695

20. Ellis J. The impact of lung cancer on patients and carers. Chron Respir Dis. 2012;9(1):39-47. http://dx.doi.org/10.1177/1479972311433577

21. Glajchen M. Physical well-being of oncology caregivers: an important quality-of-life domain. Semin Oncol Nurs. 2012;28(4):226-35. http:// dx.doi.org/10.1016/j.soncn.2012.09.005

22. Nijboer $C$, Tempelaar $R$, Sanderman R, Triemstra M, Spruijt RJ, van den Bos GA. Cancer and caregiving: the impact on the caregiver's health. Psychooncology. 1998;7(1):3-13. http://dx.doi.org/10.1002/ (SICI)1099-1611(199801/02)7:1<3::AID-PON320>3.0.CO;2-5

23. O'Hara RE, Hull JG, Lyons KD, Bakitas M, Hegel MT, Li Z, et al Impact on caregiver burden of a patient-focused palliative care intervention for patients with advanced cancer. Palliat Support Care. 2010;8(4):395-404. http://dx.doi.org/10.1017/S1478951510000258

24. Harding R, Higginson IJ, Donaldson N. The relationship between patient characteristics and carer psychological status in home palliative cancer care. Support Care Cancer. 2010:11(10):638-43. http://dx.doi.org/10.1007/s00520-003-0500-6

25. Burridge LH, Barnett $A G$, Clavarino AM. The impact of perceived stage of cancer on carers' anxiety and depression during the patients' final year of life. Psychooncology. 2009;18(6):615-23. http:// dx.doi.org/10.1002/pon.1435

26. National Alliance for Caregiving; American Association of Retired Persons Public Policy Institute. Caregiving in the U. S. 2015 Report. Chicago (IL): University of Illinois; 2015.

27. Murray SA, Kendall M, Boyd K, Sheikh A. Illness trajectories and palliative care. BMJ. 2005;330(7498):1007-11. http://dx.doi. org/10.1136/bmj.330.7498.1007

28. Murray SA, Kendall M, Boyd K, Grant L, Highet G, Sheikh A Archetypal trajectories of social, psychological, and spiritua wellbeing and distress in family care givers of patients with lung cancer: secondary analysis of serial qualitative interviews. BMJ. 2010;340:c2581. http://dx.doi.org/10.1136/bmj.c2581

29. Valeberg BT, Grov EK. Symptoms in the cancer patient: of importance for their caregivers' quality of life and mental health? Eur J Onco Nurs. 2013;17(1):46-51. http://dx.doi.org/10.1016/j.ejon.2012.01.009 